DOI: https://doi.org/10.11144/Javeriana.upsy19.epbi

\title{
Percepción subjetiva de manejo emocional, ansiedad y patrones de ingesta relacionados con aislamiento por COVID-19 *
}

Subjective Perception of Emotional Management, Anxiety and Intake Patterns Related to Isolation by COVID-19

\author{
TANiA YAdira Martínez RodríGuez ${ }^{\mathrm{a}}$ \\ Universidad de Guadalajara, México \\ ORCID: https://orcid.org/0000-0002-0456-3471 \\ Samantha Josefina Bernal-Gómez \\ Universidad de Guadalajara, México \\ ORCID: https://orcid.org/0000-0002-8039-0015 \\ Ana Paola Mora Vergara \\ Universidad de Guadalajara, México \\ ORCID: https://orcid.org/0000-0001-8319-7563 \\ Nelson Eduardo Hun Gamboa \\ Universidad de Guadalajara, México \\ ORCID: https://orcid.org/0000-0002-7614-9990
}

a Autor de correspondencia. Correo electrónico: tymartinezr@gmail.com

Para citar este artículo: Martínez Rodríguez, T. Y., Bernal-Gómez, S. J., Mora Vergara, A. P., \& Hun Gamboa, N. E. (2020). Percepción subjetiva de manejo emocional, ansiedad y patrones de ingesta relacionados con aislamiento por COVID-19. Universitas Psychologica, 19, 1-9. https://doi.org/10.1 1144/Javeriana.upsy19.epbi

\section{RESUMEN}

El periodo de aislamiento social se considera un detonante de ansiedad, y genera alteraciones en la forma de alimentarse. El objetivo de esta investigación fue determinar la relación entre la percepción subjetiva del manejo de las emociones y la ansiedad con los patrones disfuncionales de la ingesta en el periodo de aislamiento social por COVID-19, en participantes colombianos. La investigación es exploratoria de tipo transversal analítico. Un total de 450 colombianos mayores de 18 años (sexo: $82 \%$ femenino y $18 \%$ masculino) respondieron un cuestionario virtual sobre la percepción subjetiva del manejo de las emociones y la ansiedad; también, se aplicó el cuestionario de tres factores de alimentación para identificar patrones disfuncionales de la ingesta. Se reportó un riesgo 3 veces mayor de presentar ansiedad en las personas que manifestaron un manejo inadecuado de sus emociones. Asimismo, aquellos cuya percepción fue un inadecuado manejo de emociones y ansiedad presentaron mayor nivel de patrones disfuncionales de ingesta como la desinhibición $(p<0.0001)$ e ingesta emocional $(p<0.0001)$. Se concluyó que la percepción subjetiva del manejo inadecuado de las emociones y de ansiedad, incrementan la ingesta emocional y la desinhibición, en colombianos en aislamiento social.

\section{Palabras clave}

aislamiento social; inteligencia emocional; ansiedad; restricción; desinhibición; ingesta emocional.

\footnotetext{
ABSTRACT

The period of social isolation considers an anxiety trigger and generates alterations in the way people eat. The objective of this research was to determine the relationship between subjective perception of emotion
} 
management and anxiety with dysfunctional intake patterns in the period of social isolation by COVID-19 in Colombian participants. The research is exploratory of analytical cross-sectional type. A total of 450 Colombians over 18 years old (sex: $82 \%$ female and $18 \%$ male) answered a virtual questionnaire on the subjective perception of the management of emotions and anxiety, also, the questionnaire of three feeding factors was applied to identify dysfunctional intake patterns. 3 times higher risk of presenting anxiety was reported in people who manifested a specific management of their emotions. Likewise, subjects whose perception was inadequate handling of emotions and anxiety presented higher levels of dysfunctional intake patterns such as disinhibition $(p$ $<0.0001)$ and emotional intake $(p<0.0001)$. Finally, it was concluded that the subjective perception of the management of emotions and anxiety has an association with disinhibition and emotional intake in Colombians in social isolation.

Keywords

social isolation; emotional intelligence; anxiety; restriction; disinhibition; emotional intake.

La pandemia por el virus de SARS-CoV-2, que produce la enfermedad por coronavirus COVID-19, generó diversas recomendaciones gubernamentales a nivel mundial, entre ellas el aislamiento preventivo; en Colombia, estas medidas iniciaron el mes de marzo para reducir la probabilidad de contagio y el incremento de la propagación del virus (Mera Mamián et al., 2020; Mois, 2020). Estas medidas han sido fundamentales en el manejo de otras epidemias como el síndrome respiratorio agudo grave (SARS), la influenza A (H1N1) y el síndrome respiratorio de medio oriente (MERS), por ejemplo (Wilder-Smith \& Freedman, 2020).

Si bien estas estrategias parecieran cumplir con su objetivo respecto a la curva de contagios, las repercusiones psicologícas, económicas y sociales han sido tema de interés de un sinnúmero de investigaciones. Lo anterior, debido a que estas estrategias (i. e., aislamiento social, confinamiento) tienen como resultado que gran parte de la población se encuentre en sus casas realizando actividades laborales y académicas o en un panorama desalentador en desempleo, que en el contexto de los países latinoamericanos, se identifica con una gran proporción de la población que vive de la económica informal
(Ramos Soto, 2018). Como consecuencia, el cambio repentino de actividades y la carga económica, laboral y familiar, influye en el afrontamiento de los acontecimientos de la vida cotidiana, en el cómo responden las personas a estas nuevas condiciones ambientales y en la percepción de emociones negativas, como la ansiedad, lo cual se evidencia en la salud física y psicológica de la población (Mera-Mamián et al., 2020). Por esto, ante la actual crisis de salud, es primordial el cuidado de los aspectos psicoemocionales, como prevención de la disfunción emocional en el aislamiento preventivo (Mois, 2020).

Las emociones son reacciones cognitivas, fisiológicas y conductuales frente a estímulos internos o ambientales que influyen en la conducta del individuo. El procesamiento de las emociones depende de la percepción subjetiva de las mismas y la interpretación que el organismo en cuestión haga de ello, así como de la experiencia y las estrategias de afrontamiento que haya adquirido (Mois, 2020). No obstante, esta percepción no constituye un estado en el que el sujeto se encuentre pasivo, sino que, por el contrario, es un fenómeno activo e intrínsecamente cognitivo y conductual con consecuencias emocionales distintas; así pues, es propia de cada sujeto (Melamed, 2016), por lo que las respuestas de afrontamiento son indivuales y particulares, es decir, en situaciones específicas (Macías et al., 2013). Adicionalmente, un factor implicado en este proceso es la inteligencia emocional, conceptualizada como las predisposiciones individuales con identificación efectiva y regulación de las emociones (Morales Rodríguez, 2018; Pucci et al., 2019; Zysberg, 2018).

Dependiendo de la interpretación y experiencia del organismo implicado, se presentan diferentes tipos de emociones; sin embargo, son de interés en este periodo (i. e., aislamiento), las que generan malestar al organismo, denominadas emociones negativas. En la clasificación de emociones negativas, es posible identificar la ansiedad, que es una reacción emocional displacentera de carácter 
anticipatorio (Sierra et al., 2003), que puede incrementar el nivel de distintos patrones disfuncionales de la ingesta como: (1) la restricción cognitiva, que se refiere a una restricción voluntaria de la ingesta con el fin de no aumentar de peso; (2) la desinhibición, que se relaciona con los episodios de sobrealimentación y (3) la ingesta emocional, que se da en respuesta a emociones negativas (Cardi et al., 2015; Lavender et al., 2013). Lo anterior, en el contexto de la alimentación emocional, que consiste en afrontar las emociones negativas mediante el consumo de alimentos como un distractor o como estrategia de enmascaramiento de dichas emociones (Bennett et al., 2013).

Las consecuencias de la alimentación emocional suelen relacionarse con el incremento del peso corporal, lo que a su vez aumenta el riesgo de desarrollo de patologías, como las enfermedades crónico-degenerativas o los trastornos de la conducta alimentaria. Considerando las altas cifras de exceso de peso en Colombia (56.5\%), es probable que ante la situación actual (i. e., aislamiento por pandemia) e incremento de la ansiedad, también aumente la alimentación emocional (Devonport et al., 2019). Debido a ello, el objetivo de esta investigación fue determinar la relación entre la percepción subjetiva del manejo de las emociones y la ansiedad con los patrones disfuncionales de la ingesta en participantes colombianos, durante el periodo de aislamiento social por COVID-19.

\section{Método}

\section{Participantes e instrumentos}

Se realizó una investigación exploratoria de tipo transversal analítico, mediante el envío de un cuestionario virtual por redes sociales disponible durante todo el mes de abril de 2020. El muestreo fue no probabilístico con técnica en bola de nieve. En los criterios de inclusión, se tuvieron: la aceptación de participación en la investigación, la mayoría de edad ( $>18$ años), encontrarse en aislamiento social y residir en Colombia. En total, se obtuvieron 450 respuestas. Se realizaron preguntas: 1) sociodemográficas y de percepción subjetiva de la ansiedad mediante la pregunta: “iha sentido ansiedad en este periodo?"; 2) de percepción subjetiva del manejo de las emociones con la pregunta: "iconsidera que ha podido manejar sus emociones en este periodo?" y 3) la realización de alguna dieta previa a este periodo con la pregunta: "iantes del aislamiento social, llevaba alguna dieta?", todas con opción de respuesta sí/no. También, fue aplicado el cuestionario de tres factores de alimentación 18 ítems, validado en su versión en español por Jáuregui-Lobera et al. (2014), para identificar los niveles de restricción cognitiva, desinhibición e ingesta emocional.

\section{Análisis estadístico}

Las variables sociodemográficas fueron analizadas mediante distribución de frecuencias. Para definir la normalidad de la muestra se aplicó la prueba de Kolmogorov-Smirnov, obteniendo un comportamiento no paramétrico. Por lo tanto, se realizaron las pruebas de Mann Whitney para comparación entre dos grupos, Kruskall Wallis para comparación entre varios grupos no pareados y el test post hoc de Dunn. Se aplicó la prueba chi cuadrado para evaluar la asociación entre la percepción del manejo de emociones y la ansiedad, y entre la dieta previa al periodo de aislamiento y la ansiedad. El nivel de confianza fue del $95 \%$, y todas las pruebas estadísticas se realizaron a dos colas en el programa estadístico GraphPad Prism.

\section{Consideraciones éticas}

Debido a que la presente investigación no tiene riesgo ético de acuerdo a la norma oficial mexicana NOM-012-SSA3-2012, artículo 23, la carta de consentimiento informado no es un requisito para solicitar la autorización del proyecto o protocolo de investigación. Sin embargo, se tomaron como críterios para los procedimientos, lo estipulado en la declaración de Helsinki. Los participantes aceptaron su participación de manera voluntaria mediante un 
consentimiento virtual, en el que se indicó el objetivo del estudio, la libertad de retirar su consentimiento, la garantía de recibir respuesta y aclaración a cualquier duda.

\section{Resultados}

\section{Variables sociodemográficas}

En esta investigación participaron 450 colombianos. El $70.2 \%$ de los participantes fueron adultos jóvenes entre 18 y 35 años y el $82 \%$ de sexo femenino. En cuanto al nivel educativo la mayor participación (43\%) fue de profesionales con título universitario (Tabla 1$)$.

\section{Tabla 1}

Características sociodemográficas de la población

\begin{tabular}{lcc}
\hline \multicolumn{1}{c}{ Variable } & $\boldsymbol{n}$ & $\mathbf{\%}$ \\
\hline Edad & 316 & 70.2 \\
$18-35$ & 126 & 28 \\
$36-59$ & 8 & 1.8 \\
$>60$ & & \\
Sexo & 367 & 82 \\
$\quad$ Femenino & 83 & 18 \\
$\quad$ Masculino & & \\
Nivel educativo & 26 & 6 \\
$\quad$ Secundaria & 105 & 23 \\
Estudiante universitario & 193 & 43 \\
Professional & 126 & 28 \\
$\quad$ Posgrado &
\end{tabular}

Nota. Los porcentajes se establecieron a partir del total de la muestra.

Patrones disfuncionales de la ingesta en relación con el sexo, el nivel educativo y la edad

En esta investigación, se evidenció mayor nivel de ingesta emocional en el sexo femenino $(p$ $=0.025)$, no se observaron diferencias en la restricción cognitiva y desinhibición entre ambos sexos (Tabla 2).
Tabla 2

Patrones disfuncionales de la ingesta en relación con el sexo

\begin{tabular}{lrrr}
\hline $\begin{array}{c}\text { Patrones } \\
\text { disfuncionales } \\
\text { de la ingesta }\end{array}$ & Femenino & Masculino & $\boldsymbol{p}$ \\
\hline $\mathrm{RC}$ & $14.3 \pm 3.55$ & $13.49 \pm 3.41$ & 0.083 \\
$\mathrm{DES}$ & $17.67 \pm 5.46$ & $17.31 \pm 5.96$ & 0.5211 \\
$\mathrm{IE}$ & $5.88 \pm 2.5$ & $5.24 \pm 2.42$ & $0.025^{*}$ \\
\hline
\end{tabular}

Nota. Prueba Mann Whitney. * $p<$

0.05. RC: restricción cognitiva; DES:

desinhibición; IE: Ingesta emocional.

En cuanto al nivel educativo, se presentaron diferencias significativas para los tres patrones disfuncionales de la ingesta: restricción cognitiva $(p=0.0196)$, desinhibición $(p<0.0001)$ e ingesta emocional $(p=0.0005)$. Los participantes con posgrado y profesionales presentaron una mayor restricción cognitiva respecto a los estudiantes universitarios, y los estudiantes universitarios tuvieron un mayor puntaje de desinhibición e ingesta emocional en comparación con los profesionales y participantes con posgrado (Tabla 3).

\section{Tabla 3}

Patrones disfuncionales de la ingesta en relación con el nivel educativo

\begin{tabular}{lrrrr}
\hline $\begin{array}{c}\text { Patrones } \\
\text { disfuncionales } \\
\text { de la ingesta }\end{array}$ & Secundaria & $\begin{array}{c}\text { Estudiante } \\
\text { universitario }\end{array}$ & Professional & Posgrado \\
\hline RC & $13.35 \pm 3.42$ & $13.58 \pm 3.57$ & $14.12 \pm 3.38$ & $14.83 \pm 3.69^{*}$ \\
DES & $18.81 \pm 7.2$ & $19.67 \pm 5.87$ & $17.33 \pm 5.2$ & $16.07 \pm 4.86^{* * *}$ \\
IE & $5.38 \pm 2.35$ & $6.73 \pm 2.71$ & $5.54 \pm 2.41$ & $5.37 \pm 2.28^{* * *}$ \\
\hline
\end{tabular}

Nota. Prueba Kruskall Wallis para comparación entre nivel educativo. Test Dunn de comparación múltiple. ${ }^{*} p<0.05, * * * p<0.001$. RC: restricción cognitiva; DES: desinhibición; IE: Ingesta emocional. $(\mathrm{RC}=*$ Estudiante Universitario vs Posgrado; $\mathrm{DES}=* *$

Estudiante universitario vs profesional $* * *$ Estudiante Universitario vs Posgrado; IE = ** Estudiante universitario vs profesional *** Estudiante Universitario vs Posgrado).

Respecto a la edad, se observó diferencia significativa para los tres patrones disfuncionales, restricción cognitiva $(p=0.0004)$, desinhibición $(p<0.0001)$ e ingesta emocional $(p=0.0002)$. Los adultos de 36 a 59 años registraron mayor restricción cognitiva y los adultos jóvenes de 18-35 años mostraron mayor desinhibición e ingesta emocional. Asimismo, los adultos mayores, específicamente después de los 60 años, 
presentaron menor puntaje de los tres patrones disfuncionales de la ingesta (Tabla 4).

\section{Tabla 4}

Patrones disfuncionales de la ingesta en relación con la edad

\begin{tabular}{lrrr}
\hline \multirow{2}{*}{$\begin{array}{c}\text { Patrones } \\
\text { disfuncionales } \\
\text { de la ingesta }\end{array}$} & \multicolumn{3}{c}{ Edad } \\
\cline { 2 - 4 } RC & $13.78 \pm 3.51$ & $15.17 \pm 3.46$ & $12.63 \pm 2.38$ \\
DES & $18.46 \pm 5.66$ & $15.75 \pm 4.78$ & $13.13 \pm 2.53$ \\
IE & $6.07 \pm 2.62$ & $5.11 \pm 2.01$ & $3.62 \pm 1.06$ \\
\hline
\end{tabular}

Nota. Prueba Kruskall Wallis. Test Dunn de comparación múltiple. Todos los valores son significativos a $p<0.001$. Edad $(\mathrm{RC}=* * *$ $18-35$ vs 36-59 años; $\mathrm{DES}=* * * 18-35$ vs $36-59$ años * $18-35$ vs $>60$ años; $\mathrm{IE}=* *$ $18-35$ vs 36-59 años * $18-35$ vs $>60$ años).

Percepción subjetiva del manejo de las emociones y el riesgo de percibir ansiedad

En este análisis, se encontró que la razón entre la percepción subjetiva de ansiedad vs. los que no la percibieron es tres veces mayor en personas que manifestaron un manejo inadecuado de sus emociones, durante este periodo de aislamiento social, representando una asociación estadísticamente significativa $(p<$ 0.0001). Por lo tanto, la percepción de no saber manejar las emociones es un factor de riesgo para el incremento de la ansiedad en esta muestra de participantes colombianos (Figura 1).

\section{Figura 1}

Percepción subjetiva del manejo de las emociones y el riesgo de percibir ansiedad.

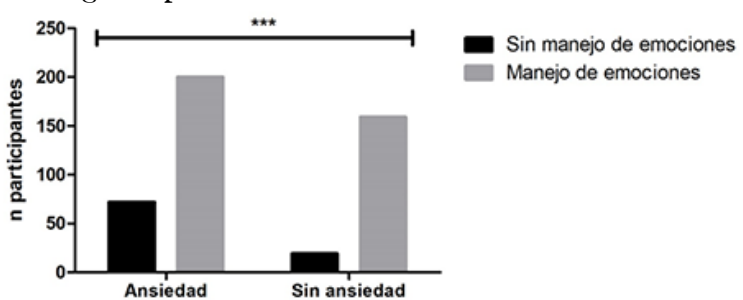

Nota. Los sujetos que no han podido manejar sus emociones tienen un riesgo tres veces mayor $(\mathrm{OR}=3, \mathrm{IC} 95 \%=1.74-5.2)$ de percibir ansiedad $(p<0.0001)$. Prueba chi cuadrado.

Percepción subjetiva del manejo de las emociones, la ansiedad y la dieta previa al aislamiento social en relación con los patrones disfuncionales de la ingesta

En cuanto a la percepción del manejo de las emociones, las personas que manifestaron un manejo inadecuado de sus emociones en este periodo presentaron mayor desinhibición $(p<$ 0.0001) e ingesta emocional $(p<0.0001)$, respecto de las que sí han podido manejar sus emociones (Tabla 5). Por otro lado, en esta misma Tabla 5, se puede observar que los participantes que manifestaron presentar ansiedad en este periodo de aislamiento social obtuvieron un mayor puntaje de desinhibición $(p<0.0001)$ e ingesta emocional $(p<0.0001)$, y que haber realizado dieta previamente al aislamiento social se asoció de manera significativa con la restricción cognitiva $(p=0.269)$. La dieta previa al aislamiento no se asoció con la percepción subjetiva de ansiedad durante el aislamiento social $(\mathrm{OR}=1.45$, IC $95 \%=0.89-2.37, p=$ 0.1473).

\section{Tabla 5}

Percepción subjetiva del manejo de emocional, ansiedad y dieta previa al aislamiento en relación a los patrones disfuncionales de la ingesta

\begin{tabular}{|c|c|c|c|}
\hline $\begin{array}{c}\text { Patrones } \\
\text { disfuncionales } \\
\text { de la ingesta }\end{array}$ & $\begin{array}{c}\text { Manejo } \\
\text { emocional } \\
(n=359)\end{array}$ & $\begin{array}{c}\text { Sin manejo } \\
\text { emocional } \\
(n=91)\end{array}$ & $p$ \\
\hline $\mathrm{RC}$ & $14.19 \pm 3.57$ & $13.98 \pm 3.43$ & 0.5997 \\
\hline DES & $16.69 \pm 5.12$ & $21.21 \pm 5.72$ & $<0.0001^{* * * *}$ \\
\hline IE & $5.35 \pm 2.32$ & $7.38 \pm 2.54$ & $<0.0001^{* * *}$ \\
\hline $\begin{array}{c}\text { Patrones } \\
\text { disfuncionales } \\
\text { de la ingesta }\end{array}$ & $\begin{array}{c}\text { Ansiedad } \\
(n=272)\end{array}$ & $\begin{array}{l}\text { Sin ansiedad } \\
\quad(n=178)\end{array}$ & $p$ \\
\hline $\mathrm{RC}$ & $14.14 \pm 3.32$ & $14.16 \pm 3.85$ & 0.9348 \\
\hline DES & $19.69 \pm 5.27$ & $14.44 \pm 4.35$ & $<0.0001 * * *$ \\
\hline IE & $6.59 \pm 2.49$ & $4.49 \pm 1.9$ & $<0.0001^{* * *}$ \\
\hline $\begin{array}{c}\text { Patrones } \\
\text { disfuncionales } \\
\text { de la ingesta }\end{array}$ & $\begin{array}{c}\text { Dieta previa } \\
\text { al aislamiento } \\
(n=89)\end{array}$ & $\begin{array}{l}\text { Sin dieta previa } \\
\text { al aislamiento } \\
(n=361)\end{array}$ & 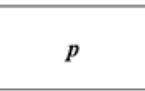 \\
\hline $\mathrm{RC}$ & $14.83 \pm 3.2$ & $13.98 \pm 3.6$ & $0.0269^{*}$ \\
\hline DES & $18.21 \pm 6.06$ & $17.46 \pm 5.42$ & 0.3893 \\
\hline IE & $6.03 \pm 2.6$ & $5.7 \pm 2.47$ & 0.286 \\
\hline
\end{tabular}

\section{Discusión}

En esta investigación, se encontró mayor nivel de ingesta emocional en los participantes de sexo femenino, lo cual coincide con los resultados 
de estudios donde se presentó una relación directa entre el nivel de estrés y la alimentación emocional, y se obtuvieron puntuaciones más altas en alimentación emocional en mujeres (Poínhos et al., 2013; Sanlier et al., 2015). No obstante, se presentaron algunas limitaciones que pueden tenerse en cuenta en posteriores investigaciones, al ser un muestreo no probabilístico la mayor participación fue de sexo femenino, similar a otras investigaciones donde más del $70 \%$ corresponde a mujeres (Bourdier et al., 2018; Penaforte et al., 2019; Poínhos et al., 2013; Sanlier et al., 2015).

En cuanto al nivel educativo, las personas con posgrado o profesionales mostraron mayor nivel de restricción cognitiva, que se podría explicar por su nivel de conocimiento, la asociación con una mayor resistencia emocional, niveles más bajos de patologías y mejores posibilidades de recuperación de traumas o situaciones estresantes (Zysberg \& Rubanov, 2010). Se proponen nuevas investigaciones con poblaciones con un rango académico y de edad similar, ya que este estudio muestra heterogeneidad en la muestra.

En el presente trabajo, se observó que los estudiantes universitarios colombianos tienen una mayor tendencia a la desinhibición o sobrealimentación y a presentar ingesta emocional, que se puede relacionar con el estado de estrés y ansiedad que genera la vida universitaria, el cambio inesperado a la educación virtual y el aislamiento social por la contingencia presentada; esto es similar a los hallazgos de un estudio en universitarios de Turquía, en el cual también se observó mayor desinhibición e ingesta emocional (Sanlier et al., 2015), sin otro estresor como el actual que es el aislamiento social, situación que podría exacerbar aún más estos patrones disfuncionales. No obstante, hay resultados opuestos en investigaciones realizadas en países europeos, donde han encontrado incremento de los tres patrones disfuncionales de la ingesta (Poínhos et al., 2013). Sin embargo, el contexto social de los países latinoamericanos, como Colombia, es diferente, y además no hay evidencia de estos aspectos en una situación similar a la pandemia por COVID-19.

Además, los adultos jóvenes de 18 a 35 años también presentaron mayor nivel de desinhibición en ingesta emocional, lo cual, al realizar un contraste en conjunto con el aspecto educativo, muestra que los estudiantes universitarios generalmente se encuentran en dichos rangos de edad. De igual manera, los adultos entre 36 y 59 años presentaron mayor restricción cognitiva y, teniendo en cuenta que es el rango de edad en el que generalmente se encuentran los profesionales o personas con posgrado, los resultados son acordes y serían evidencia nueva al respecto. Es recomendable incluir a futuro para el caso de estudiantes universitarios, variables como el tipo de pregrado o licenciatura y el último semestre cursado, ya que se pueden obtener hallazgos novedosos al respecto de los patrones disfuncionales de la ingesta. Adicionalmente, para el caso de las personas con posgrado, es importante conocer el tipo de posgrado y su ocupación actual.

La percepción de manejo de emociones es fundamental para reconocer el estilo de afrontamiento ante situaciones estresantes y el correcto reconocimiento de las emociones para responder ante una situación específica (Macías et al., 2013), por esta razón, la inteligencia emocional es un recurso de afrontamiento, identificándose que las personas que manifestaron no poder manejar sus emociones en este periodo presentaron un riesgo tres veces mayor de percibir ansiedad. Esto se relaciona y conincide con el argumento de que la inteligencia emocional está relacionada de manera negativa con la alimentación emocional (Zysberg \& Rubanov, 2010).

De igual manera, los participantes que manifestaron no saber manejar sus emociones presentaron mayor desinhibición e ingesta emocional, lo cual es acorde a lo expuesto en el modelo de la neurociencia afectiva (Silva, 2008a, 2008b; Silva et al., 2002a, 2002b), que explica que, ante un estímulo estresante, los comedores emocionales tienden a la desinhibición o sobrealimentación. En 
esta investigación, esta situación parece ser generalizada para los participantes colombianos.

Adicionalmente, se observó que la percepción subjetiva de la ansiedad durante el aislamiento social, también se asoció con mayor nivel de desinhibición e ingesta emocional. Este resultado se relaciona con los obtenidos en otras investigaciones, en las que han demostrado que la ansiedad media la asociación entre la inteligencia emocional y los patrones disfuncionales de la ingesta (Zysberg, 2018) y tiene una asociación negativa con la alimentación (Bennett et al., 2013).

En el modelo de la neurociencia afectiva, se establece que los sujetos que realizan dietas son comedores emocionales, y tienen un mayor nivel tanto de restricción como de desinhibición (Silva, 2008a), por lo que en la presente investigación se indagó al respecto, preguntando sobre dieta previa al periodo de aislamiento, y como era de esperarse, al ser las dietas de características restrictivas, esta variable se relacionó de manera significativa con mayor puntuación de restricción cognitiva $(p=$ 0.0269) y un leve incremento en el puntaje de desinhibición e ingesta emocional, que no fue estadísticamente significativo. Por esta razón, se requiere más investigación y profundización en los aspectos relacionados con el historial de dietas restrictivas.

Este trabajo es evidencia novedosa sobre la inteligencia emocional y el enmascaramiento de las emociones con la ingesta de alimentos, en este periodo que incrementa la ansiedad y que se caracteriza por ser único en las últimas décadas. Se deben tener en cuenta las limitaciones de este estudio exploratorio, las cuales ya han sido mencionadas a lo largo de la discusión y otras variables que no se pueden controlar por el tipo de investigación, como lo son las genéticas, epigenéticas y fisiológicas; además, es importante reconocer la heterogeneidad y diversidad de la muestra de esta investigación y se recomienda más investigación especifica por grupo poblacional.

\section{Conclusión}

Por último, se concluye que los participantes colombianos cuya percepción es un manejo inadecuado de sus emociones, presentan un riesgo mayor de percibir ansiedad, y a su vez, se relaciona con un incremento en la desinhibición e ingesta emocional durante el periodo evaluado de aislamiento social por COVID-19.

\section{Agradecimientos}

Agradecemos a todas las personas, empezando por los voluntarios para el estudio y todas las personas que de una u otra forma han contribuido al desarrollo de este trabajo, y al Instituto de Investigaciones en Comportamiento Alimentario y Nutrición (IICAN) de la Universidad de Guadalajara (UdeG).

\section{Referencias}

Bennett, J., Greene, G., \& Schwartz-Barcott, D. (2013). Perceptions of emotional eating behavior. A qualitative study of college students. Appetite, 60(1), 187-192. https:// doi.org/10.1016/j.appet.2012.09.023

Bourdier, L., Morvan, Y., Kotbagi, G., Kern, L., Romo, L., \& Berthoz, S. (2018). Examination of emotion-induced changes in eating: A latent profile analysis of the Emotional Appetite Questionnaire. Appetite, 123, 72-81. https://doi.org/10.101 6/j.appet.2017.11.108

Cardi, V., Leppanen, J., \& Treasure, J. (2015). The effects of negative and positive mood induction on eating behaviour: A metaanalysis of laboratory studies in the healthy population and eating and weight disorders. Neuroscience and Biobehavioral Reviews, 57, 299-309. https://doi.org/10.1016/j.neubiore v.2015.08.011

Devonport, T. J., Nicholls, W., \& Fullerton, C. (2019). A systematic review of the association between emotions and eating behaviour in normal and overweight adult 
populations. Journal of Health Psychology, 24(1), 3-24. https://doi.org/10.1177/13591 05317697813

Jáuregui-Lobera, I., García-Cruz, P., CarboneroCarreño, R., Magallares, A., \& RuizPrieto, I. (2014). Psychometric properties of Spanish version of the three-factor eating questionnaire-R18 (Tfeq-Sp) and its relationship with some eating- and body image-related variables. Nutrients, 6(12), 5619-5635. https://doi.org/10.3390/nu612 5619

Lavender, J. M., De Young, K. P., Wonderlich, S. A., Crosby, R. D., Engel, S. G., Mitchell, J. E., Crow, S. J., Peterson, C. B., \& Le Grange, D. (2013). Daily patterns of anxiety in anorexia nervosa: Associations with eating disorder behaviors in the natural environment. Journal of Abnormal Psychology, 122(3), 672-683. https://doi.org /10.1037/a0031823

Macías, M., Madariaga-Orozco, C., ValleAmarís, M., \& Zambrano, J. (2013). Estrategias de afrontamiento individual y familiar frente a situaciones de estrés psicológico Individual and family copying strategies when facing psychological stress situations. Psicología desde El Caribe, 30(1), 123-145. https://www.redalyc.org/pdf/213/ 21328600007.pdf

Melamed, A. (2016). Las teorías de las emociones y su relación con la cognición: un análisis desde la filosofía de la mente. Cuadernos de La Facultad de Humanidades y Ciencias Sociales, 49, 13-38. https://www.redalyc.org /pdf/185/18551075001.pdf

Mera Mamián, A. Y., Tabares González, E., Montoya González, S., Muñoz Rodríguez, D. I., \& Monsalve Vélez, F. (2020). Recomendaciones prácticas para evitar el desacondicionamiento físico durante el confinamiento por pandemia asociada a COVID-19. Universidad y Salud, 22(2), 166-177. https://doi.org/10.22267/rus.2022 02.188

Mois, J. M. (2020). Aspectos psicologicos del COVID-19. Universidad Internacional de La Rioja. Editorial Tektime
Morales Rodríguez, F. M. (2018). Estrategias de afrontamiento en una muestra de estudiantes universitarios. International Journal of Developmental and Educational Psychology, 2(1), 289-294. https://doi.org/1 0.17060/ijodaep.2018.n1.v2.1228

Penaforte, F. R. de O., Minelli, M. C. S., Rezende, L. A., \& Japur, C. C. (2019). Anxiety symptoms and emotional eating are independently associated with sweet craving in young adults. Psychiatry Research, 271, 715-720. https://doi.org/10.1016/j.psy chres.2018.11.070

Poínhos, R., Oliveira, B. M. P. M., \& Correia, F. (2013). Eating behaviour patterns and BMI in Portuguese higher education students. Appetite, 71, 314-320. https://doi.org/10.10 16/j.appet.2013.08.024

Pucci, M., Di Bonaventura, M. V. M., Vezzoli, V., Zaplatic, E., Massimini, M., Mai, S., Sartorio, A., Scacchi, M., Persani, L., Maccarrone, M., Cifani, C., \& D'Addario, C. (2019). Preclinical and clinical evidence for a distinct regulation of $\mathrm{Mu}$ Opioid and type 1 Cannabinoid receptor genes expression in obesity. Frontiers in Genetics, 10, 1-14. https://doi.org/10.3389/fgene.201 9.00523

Ramos Soto, A. L. (2018). El papel del sector informal en dos economías de América Latina\#: México y Guatemala. Revista Iberoamericana de Contaduría, Economía y Administración, 7(14), 1-25. https://doi.org/ 10.23913/ricea.v7i14.118

Sanlier, N., Biyikli, A. E., \& Biyikli, E. T. (2015). Evaluating the relationship of eating behaviors of university students with body mass index and self-esteem. Ecology of Food and Nutrition, 54(2), 175-185. https:// doi.org/10.1080/03670244.2014.896798

Sierra, J. C., Ortega, V., \& Zubeidat, I. (2003). Ansiedad, angustia y estrés: tres conceptos a diferenciar. Revista Mal-estar e Subjetividade, 3(1), 10-59. https://www.reda lyc.org/articulo.oa?id $=27130102$

Silva, J. R. (2008a). Overeating and restrained eaters. An affective neuroscience perspective. Revista Medica de Chile, 136, 
1336-1342. http://www.scopus.com/inward /record.url?eid=2-s2.0-64249108786\&par tnerID $=40 \&$ md5 $=\mathrm{c} 19 \mathrm{c} 3677 \mathrm{e} 98837 \mathrm{e} 2 \mathrm{dd}$ $60 \mathrm{abadc} 012 \mathrm{~d} 203$

Silva, J. R. (2008b). Sobrealimentación inducida por la ansiedad. Parte II\#: Un marco de referencia neurocientífico para el desarrollo de técnicas psicoterapéuticas y programas de prevención. Terapia Psicológica, 26(1), 99-115. https://dialnet.unirioja.es/servlet/a rticulo? codigo $=2694390$

Silva, J. R., Pizzagalli, D. A., Larson, C. L., Jackson, D. C., \& Davidson, R. J. (2002a). Frontal brain asymmetry in restrained eaters. Journal of Abnormal Psychology, 111(4), 676-681. https://doi.org/10.1037//0 021-843X.111.4.676

Silva, J. R., Pizzagalli, D. A., Larson, C. L., Jackson, D. C., \& Davidson, R. J. (2002b). Problematic eating behaviors and nutritional status in 7 to 12 yearold Chilean children. Journal of Abnormal Psychology, 111(4), 676-681. https://doi.org /10.1016/S1697-2600(13)70005-X

Wilder-Smith, A., \& Freedman, D. O. (2020). Isolation, quarantine, social distancing and community containment: Pivotal role for old-style public health measures in the novel coronavirus (2019-nCoV) outbreak. Journal of Travel Medicine, 27(2), 1-4. https ://doi.org/10.1093/jtm/taaa020

Zysberg, L. (2018). Emotional intelligence, anxiety, and emotional eating: A deeper insight into a recently reported association? Eating Behaviors, 29(2017), 128-131. https: //doi.org/10.1016/j.eatbeh.2018.04.001

Zysberg, L., \& Rubanov, A. (2010). Emotional intelligence and emotional eating patterns: A new insight into the antecedents of eating disorders? Journal of Nutrition Education and Behavior, 42 (5), 345-348. htt ps://doi.org/10.1016/j.jneb.2009.08.009

\section{Notas}

* Artículo de investigación. Los autores de esta publicación reciben beca doctoral por el Consejo Nacional de Ciencia y Tecnología
(CONACyT). TM 761992; SB 788659; AM 734107, NH 734376 (las abreviaturas de las becas doctorales, corresponden a las iniciales del nombre del autor beneficiario). 